\title{
Protective effect of supportive family relationships and the influence of stressful life events on adolescent adjustment
}

\author{
Alfredo Oliva, Jesús M. Jiménez-Morago and Águeda Parra \\ Developmental and Educational Psychology, University of Seville, Seville, Spain
}

(Received 07 May 2007; final version received 26 March 2008)

\begin{abstract}
The purpose of this study was to determine the association between the occurrence of stressful life events and internalizing and externalizing problems, and to analyze longitudinally buffering effects of supportive family relationships. To this end, 100 Spanish adolescents were studied twice, when they were in mid-adolescence (15-16 years) and two years later. They completed questionnaires regarding stressful life events, family relationships, and adolescent adjustment. Results showed that high quality parent-adolescent relationships protected boys and girls against the negative consequences of stressful life events on externalizing, but not internalizing, symptoms. The adolescents who enjoyed good relationships with their parents in mid-adolescence did not increase their externalizing problems in late adolescence as consequence of the occurrence of stressful events. However, these stressors did lead to an increase in the number of externalizing problems when the family relationships were of a middle or low quality. These results highlight the important role that supportive family relationships play in the behavioral adjustment of adolescents, protecting them against some negative consequences of stressful life events, and suggest the relevance of supporting parents through resources such as parent education in order to help them to improve their relationships with their adolescents.
\end{abstract}

Keywords: adolescence; stressful life events; externalizing and internalizing problems; family relationships; moderation

\section{Introduction}

\section{Adolescence, stressors and adjustment}

Adolescence is a developmental stage in which boys and girls must face many physical and psychological changes and deal with several developmental tasks, therefore some emotional and behavioral problems may frequently arise. Moreover, the occurrence of potentially stressful circumstances may result in additional difficulties that exceed the coping skills of the adolescent and hinder a healthy development, thereby increasing the adolescent's vulnerability to maladjustment.

Recent reviews of literature have pointed out strong evidence of the relationship between stressors, and child and adolescent psychopathology (Grant, Compas, Stuhlmacher, Thurm, McMahon, \& Halpert, 2003; Grant, Compas, Thurm, McMahon, $\&$ Gipson, 2004). The research indicates that the risk for emotional problems increases in response to the experience of stressful life events (Aseltine, Gore, \& Colten, 1994; Ge, Lorenz, Conger, Elder, \& Simmons, 1994; Kim, Conger, Elder, \& Lorenz, 2003; Kraaij 
et al., 2003; Leadbeater, Kuperminc, Blatt, \& Hertzog, 1999). There is less evidence for a link between an adversity in life circumstances and adolescent externalizing problems, such as substance abuse or aggressive and antisocial behavior, but a few cross-sectional (Jackson \& Warren, 2000; Rafnsson, Jonsson, \& Windle, 2006) and longitudinal studies (Gest, Neeman, Hubbard, Masten, \& Tellegen, 1993; Hoffman \& Su, 1997; Kim et al., 2003) have found empirical support for this relationship. Associations are typically weaker with externalizing than with internalizing problems, and some studies have failed to find a significant prospective link between stressful life events and increased externalizing symptoms (Grant et al., 2004). In summary, prospective studies on the influence of stressful events on symptomatology have provided non-conclusive results. Moreover, the proportion of variance accounted for by stressful events tends to be less than $15 \%$, probably due to certain factors, such as social support or coping strategies, which might moderate this influence (Seiffge-Krenke, 2000).

In the transactional model proposed by Lazarus and Folkman (1984), stress occurs when a relationship between the individual and the environment is perceived as exceeding his/her resources. Thus, it is the appraisal of a potential stressor which determines the level of distress that the individual experiences. This assessment, occurring from the interaction between environmental demands and the perceptions of available resources by an individual may be influenced by both internal and external factors of the individual. Among such interactions, social support appears most frequently in the literature to influence stress-related appraisals and coping strategies (Cohen \& Wills, 1985). For instance, individuals who experience increased support from their family and friends rely more on approach-coping, such as positive appraisal and seeking guidance and support, and less on avoidance-coping (Calvete \& Connor-Smith, 2006; Holahan, Moss, Holahan, \& Brennan, 1997; Tao, Dong, Pratt, Hunsberger, \& Pancer, 2000). Therefore, family relationships characterized by parental support and affection, close emotional links or authoritative parenting, can be considered a protective factor against the negative consequences of stressors on adolescent adjustment.

\section{Family relationships as a protective factor}

There is considerable evidence regarding the direct association (i.e., the main effect model) between quality family relationships and adolescent emotional and behavioral adjustment (Collins \& Laursen, 2004; Steinberg \& Silk, 2002). This main effect model indicates that social support has a positive influence on the individual, independent of experiencing stressful events. The second model is referred to as the stress-buffering model, and posits that social support, or other variables such as coping strategies, moderates the impact of stressful events on adjustment and health (Cohen \& Wills, 1985). According to the stressbuffering model, boys and girls who experience a high number of stressors, but enjoy supportive family relationships, would not show psychological maladjustment because such adolescents will make appraisals of the stressors less negative and will use improved coping strategies (Jose \& Kilburg III, 2007). Most studies that have analyzed the protective effect of social support on adolescent adjustment have jointly considered different sources of support, such as family, peers, and teachers. These studies, however, have frequently utilized a superficial assessment for each source, with scales in which only two or three items were related to parental support (Dumont \& Provost, 1999; Gore \& Aseltine, 1996 ). There are but a few studies which focus on family support and analyze family relationships in depth (Murberg \& Bru, 2004). 
Considering the research available on the protective role of quality family relationships, we have drawn several conclusions, as follows. First, these studies have focused more on the protective effects of emotional adjustment and the studies which have focused on behavioral adjustment are less frequent. Second, the results have been inconsistent, since while some studies have not found support for a buffering effect (Gore \& Aseltine, 1995; Herman-Stahl \& Petersen, 1999; Murberg \& Bru, 2004), other studies have found such an effect (DuBois, Felner, Meares, \& Kries, 1994). In any case, there exists more empirical support for a main effect model than for a buffering model, likely because interaction effects are usually small in magnitude and difficult to detect using variable-based analyses (Luthar \& Zelazo, 2003; McClelland \& Judd, 1993).

Finally, most studies with adolescents have focused on early adolescence. The research analyzing the protective effect of family relationships on late adolescence has been scarce (Luthar, 2006) and the few studies carried out have demonstrated less parental influence because during this period, the family competes with other sources of support that become more relevant, such as friends or boyfriends/girlfriends (Helsen, Vollebergh, \& Meeus, 2000; Lamothe et al., 1995; Scholte, Van Lieshout, \& Van Aken, 2001; Tao et al., 2000). Moreover, it should be pointed out that most of the research has been conducted in North America, Australia, the UK, Holland, and other North European countries. These countries show an individualistic orientation that places a high value in agency and autonomy of the adolescent. In contrast, in Spain, like in other collectivist societies, family relationships are characterized by interdependence, and family of origin plays an important role in the individual's development, which tends to keep close emotional links with the parents through adolescence and adulthood (Kagitcibasi, 1996). Therefore, it is probable that the quality of relationships within the family continues having a beneficial effect on psychological adjustment of Spanish adolescents to a later chronologic age.

Consequently, there are several reasons that justify this study. There are many findings supporting the role of the family in the protection of the emotional health of children and young adolescents facing adversity, but the evidence is reduced when analyzing the protection of behavioral adjustment during late adolescence. Moreover, research carried out in countries with a collectivist orientation, such as Spain, is scarce, thus granting additional value to this study.

Although transactional models consider stress as a relationship between environmental events or conditions and an individual's cognitive appraisals of the event or condition (Lazarus \& Folkman, 1984), we decided to use a definition of stress-based exclusively on objective environmental changes or conditions in order to detect interaction effects more precisely (Grant et al., 2003; Rice, 1999; Rudolph \& Hammen, 1999). In this model of stress, external threats to the individual represent the only element that clearly differs from other mediator or moderator factors, such as psychological symptoms or additional risk factors, thus avoiding the mix of influences that may mask interaction effects (Cohen, Kessler, \& Gordon, 1995).

The main objectives of the present study were as follows: (1) to analyze in depth the association between the occurrence of stressful life events and internalizing and externalizing problems in a sample of Spanish adolescents; and (2) to study possible main and buffering effects of quality family relationships on the emotional and behavioral consequences of stressful life events during adolescence.

The occurrence of negative events and the existence of poor family relationships in midadolescence were expected to relate to internalizing and externalizing problems and to their increase from mid-to-late adolescence. We also hypothesized that family support will protect adolescents from the potentially negative influence of stressful events. 
A. Oliva et al.

\section{Methods}

\section{Participants}

In this paper, we present a longitudinal study of a group of boys and girls followed through their adolescence. The first phase of the study was cross-sectional, in which the sample consisted of 513 Caucasian adolescents ( 221 boys and 292 girls) between 13 and 19 years of age $(M=15.43$ and $S D=1.19)$, all of whom attended private or state schools in the Spanish province of Seville. Participants were selected from a total of 10 secondary schools (six in the capital, three in rural areas, and one in the metropolitan area), chosen randomly on the basis of population size and type of school (four private and six state institutions). Seventy-four percent of the participants resided in urban areas and $26 \%$ resided in rural areas. With regard to family structure, $88 \%$ of the participants lived with their biological parents, $7 \%$ with their mother, and 3\% with their father. Only one adolescent lived in a restructured family. Fifty percent of the adolescents had a sibling, 26\% of the participants had two siblings, and $17 \%$ of the participants had three or more siblings. Seven percent of the participants were the only children in the family.

The second phase of the study consisted of following boys and girls who were at the beginning of their adolescence in the previous cross-sectional study $(N=136, M=13.11$ years, and $S D=.44)$. We followed the participants for greater than five years, until they reached 18 or 19 years of age. Thus, these teenagers completed the questionnaires at the beginning, in the middle $(M=15.38$ and $S D=.56)$, and at the end of their adolescence $(M=17.85$ and $S D=.52)$. Between each data collection, a period of time of approximately two years had elapsed.

The results presented in this paper are derived from the second and third waves of data collection, since some instruments were not applied in early adolescence. Our findings refer to middle and late adolescence and are referred to in this paper as Time 1 (T1) and Time 2 (T2), respectively.

One hundred thirty-six adolescents participated in the first wave of the study; 113 adolescents participated in the second wave (T1) and 100 participants remained in the third wave. This final sample was comprised of 38 boys and 62 girls coming from predominantly two-parent households.

Attrition analysis did not reveal significant differences between the second and the third waves, but differences were detected between the first and the third waves. In fact, our results indicate that among the adolescents that remained in the study until the end, there were more girls than boys $\left(\chi^{2}=4.05, p<.05\right)$ and less children of parents with a low level of education $\left(\chi^{2}=6.52, p<.05\right)$. However, these adolescents who participated until the end of the study were similar to those who did not continue in terms of their habitat (i.e., rural versus urban) and in terms of the educational establishment that they attended (i.e., state versus private). There were no significant differences in terms of family relationships and adolescent adjustment. Differences among schools in the variables of the study were nonsignificant.

\section{Procedure}

The goals of the study were explained to the headmasters/headmistresses of the selected schools during initial written and telephone contacts, after which an interviewer visited the school and selected the adolescents. We used a passive consent procedure. Parents were informed by mail about the study and only three parents rejected the participation of their 
children in the study. The participants completed the confidential questionnaire during a 45-minute group session, which took place on two different days.

Two and four years later, we returned to the schools to interview the selected adolescents that were still in attendance. We contacted those teenagers that were no longer in the school by phone and met them in the Faculty of Psychology, where they completed the questionnaires.

\section{Measures}

For the purposes of the study, a questionnaire was compiled which included a number of different instruments related to family relationships, stressors, and adolescent adjustment. Some of these instruments were developed ad hoc for this study, while other instruments were adaptations or translations from the works of other researchers. Table 1 includes means, $S D$ s and ranges of the study variables.

\section{Stressful life events}

Stressful life events were measured by a scale designed for this study that was applied only during T2. This scale is a self-report measure consisting of a list of 29 negative events, concerning self or significant others, likely to be experienced by adolescents in the family, school, or peer contexts (e.g., parental divorce, breaking up with boy/girlfriend, and death of a family member). These events were selected from a pilot study that we made of over 200 adolescents. In order to capture the influence of stressors over the change of internalizing and externalizing syndromes during this period, participants should indicate those events that have occurred since the last time they were interviewed. Each item was scored " 1 " if the specific event had occurred and " 0 " if the event had not occurred. A total score was composed by adding up all negative events experienced.

The Youth Self Report (YSR; Achenbach, 1991), composed by 113 items and designed to be used with adolescents between the ages of 12 and 18 years, was applied to obtain adolescent reports on their own problems. The YSR assesses two broad-band syndromes, externalizing, composed of 30 items (aggression and antisocial behavior; e.g., "I destroy things belonging to others"), and internalizing, composed of 31 items (withdrawn, somaticcomplaints, and anxiety-depression; e.g., "I feel no one loves me"). All items were rated on a three-point Likert scale where " 0 " indicates responses of "not true," " 1 " indicates "somewhat or sometimes true," and " 2 " corresponds to "very true or often true." The Spanish version of this scale has shown good reliability and validity (Abad, Forns, Amador, \& Martorell, 2000). Cronbach's alphas for this study for T1/T2 were .76/.77 for externalizing and $.89 / .86$ for internalizing problems.

\section{Parenting style}

Parenting style was assessed using an instrument consisting of 24 items which explored the adolescent's perception of the educational or disciplinary style used by his/her parents, classified according to two dimensions: acceptance/involvement and supervision/monitorization (Lamborn, Mounts, Steinberg, \& Dornbush, 1991). In the current study, the acceptance/involvement scale assesses the degree to which adolescents view their parents as being responsive and loving, and included 15 items (e.g., "I can count on her/him to help me out, if I have some kind of problem"). The supervision scale measured an adolescent's perceptions of parental monitoring and behavioral supervision (e.g., "My parents know 
Table 1. Correlations among all study variables and means, standard deviations, and range for the variables.

\begin{tabular}{lcccccccrr}
\hline & 1 & 2 & 3 & 4 & 5 & 6 & $M$ & $S D$ & Range \\
\hline 1. Stressful events & & & & & & & 6.20 & 3.17 & $0-15$ \\
2. QFR T1 & -.12 & & & & & & 32.61 & 3.70 & $17-40$ \\
3. QFR T2 & $-.32^{* *}$ & $.64^{* * *}$ & & & & & 31.40 & 3.99 & $20-46$ \\
4. Externalizing T1 & $.25^{*}$ & $-.49^{* * *}$ & $-.27^{* *}$ & & & & 13.12 & 6.72 & $1-38$ \\
5. Externalizing T2 & $.41^{* * *}$ & $-.36^{* * *}$ & $-.28^{* *}$ & $.65^{* *}$ & & & 13.62 & 6.21 & $1-31$ \\
6. Internalizing T1 & $.27^{* *}$ & -.08 & -.03 & $.38^{* * *}$ & $.31^{* *}$ & & 13.36 & 8.79 & $0-46$ \\
7. Internalizing T2 & $.29^{* *}$ & -.05 & -.11 & $.22^{*}$ & $.43^{* * *}$ & $.72^{* * *}$ & 12,29 & 7.51 & $2-38$ \\
\hline
\end{tabular}

$N=100 .{ }^{*} p<.05 ; * * p<.01 ; * * * p<.001$.

${ }^{\mathrm{a}}$ Quality of family relationships in Time 1 ; ${ }^{\mathrm{b}}$ Quality of family relationships in Time 2.

exactly where I am most afternoons after school"). The Spanish version of the scale has demonstrated concurrent and predictive validity in earlier research (Oliva \& Arranz, 2005). In the present study, internal consistency measured by Cronbach's alpha was $.68 / .76$ for acceptance and .71/.62 for supervision.

\section{Family adaptability and cohesion scale}

The Family Adaptability and Cohesion Scale (FACES II; Olson, Portner, \& Lavee, 1985) is a 30-item instrument consisting of two scales used to assess the level of cohesion and adaptability within the family: cohesion includes items about emotional bonding, supportiveness, and boundaries (e.g., "Our family does things together") and adaptability refers to leadership, discipline, roles, and rules (e.g., "In our family, it is easy for everyone to express his/her opinion"). Adolescents rated items on a five-point Likert scale. The Spanish version of FACES II and III has been validated and widely used with Spanish and South American samples (Forjaz, Martinez \& Cervera-Enguix, 2002; López, 2002; Martínez-Pampliega, Iraurgi, Galíndez \& Sanz, 2006; Zegers, Larraín, Polaino-Lorente, Trapp \& Díez, 2003). Alphas in the present study were $.84 / .87$ for cohesion and $.75 / .81$ for adaptability.

\section{Communication with parents}

This scale was elaborated ad hoc for this study and was comprised of 22 items (11 pertaining to communication with the mother and 11 pertaining to communication with the father) concerning the frequency of family speeches about topics, such as hobbies, family rules, or adolescent sexual behavior (Parra \& Oliva, 2002). Teens selected his or her answers from a scale of " $1-4$ " (i.e., I never talk to her/him, I talk rarely, I talk sometimes, or I often talk). The scale demonstrated adequate reliability in the present study, providing alpha coefficients of .80 in $\mathrm{T} 1$ and .82 in $\mathrm{T} 2$.

\section{Analysis strategy}

We had several variables that could be considered as indicators of the quality of family relationships that were highly intercorrelated. In order to avoid problems of colinearity in the regression analyses (Belsley, 1991), we performed exploratory and confirmatory factor analyses to reduce the information about family relationships. Two exploratory factor 
analyses (principal components and varimax rotation) were carried out on the following variables: acceptance (15 items), monitoring (9 items), cohesion (16 items), adaptability (14 items), and communication with parents (22 items).

Later on, two confirmatory factorial analyses using AMOS 5.0 (Arbuckle, 2003) were conducted to validate the single factor models. To test the models, beside $\chi^{2}$, well-known measures were used as goodness of fit indices (Hoyle, 1995): the root mean square error of approximation (RMSEA), the Tucker-Lewis Index (TLI) and the Comparative Fit Index (CFI).

A variable-centered approach was used to analyze moderation effects, so following the procedure proposed by Aiken and West (1991), we conducted a hierarchical regression with T2, externalizing and internalizing problems as criteria to examine the moderating effect of quality of family relationships in T1 (QFR T1). The independent (stressful life events) and moderator variables (T1 quality of family relationships) were centered, and an interaction term was created by multiplying the two centered variables. Gender was included in the first step of each regression model. Stressful life events and QFR T1 were entered in step 2 and step 3, respectively. In step 4, the interaction term (SLE $\times$ QFR T1) was added as a test of the buffering effect of quality of family relationships.

To test longitudinal effects, we employed an autoregressive model (Stoolmiller \& Bank, 1995) by means of hierarchical regressions. Externalizing problems in T1 was entered in the regression (step 1) jointly with gender (step 2), stressful life events (step 3), QFR T1 (step4), and the interaction term (step 5). Externalizing syndromes in T2 was the dependent variable. This autoregressive model converted the dependent variable into a residual change variable, and tested whether stressful events, family relationships, or their interaction, predicted an adolescent's changes in rank in externalizing problems from T1 to $\mathrm{T} 2$. The same procedure was followed with internalizing problems

\section{Results}

\section{Preliminary analysis}

The exploratory factor analyses revealed the existence of only one factor, accounting for $47.8 \%$ of the total variance in $\mathrm{T} 1$ and $53.2 \%$ of the total variance in T2. Confirmatory factor analyses carried out to test those models showed acceptable goodness of fit indices, both with the variables of $\mathrm{T} 1, \chi^{2}(5, N=100)=6,59, p=\mathrm{ns}, \mathrm{RMSEA}=.056, \mathrm{CFI}=.98$, $\mathrm{TLI}=.97$, and Time $2, \chi^{2}(5, N=100)=6,04, p=\mathrm{ns}, \quad \mathrm{RMSEA}=.046, \mathrm{CFI}=.99$, TLI $=.98$.

We named these factors "quality of family relationships in T1" (QFR T1) and "quality of family relationships in T2" (QFR T2), and we used them in the following analyses. Every factor summarized the 76 items from the three scales that assessed family relationships: parenting style, FACES and communication with parents. High scores in these factors were indicative of family relationships characterized by emotional closeness and cohesion, a non-coercive discipline, clear and flexible rules and regulations, and parenting styles based on affection, communication, and monitoring of their children's activities.

There were significant correlations between stressful life events and internalizing and externalizing syndromes, but correlations with externalizing problems were stronger, especially in T2 (Table 1). Stressful life events were also significantly correlated with QFR T2. Quality of family relationships was associated to externalizing, but not to internalizing problems. Furthermore, some correlations between these variables were significant across waves. 
The parent's level of education had no significant associations with any of the variables in the study.

\section{Family relationships as a moderator of the influence of life events on adolescent adjustment}

The next step in our analyses concerned the hypothesis that stressful life events would have a greater impact on externalizing and internalizing problems in adolescents who perceived worse family relationships, indicating that supportive parenting would protect adolescents against the negative consequences of stressors on behavior. With regard to externalizing problems, the results of the regression analysis showed that the main effects were significant, indicating that high occurrence of life events and low QFR T1 were associated with high levels of externalizing problems in T2 (Table 2). Gender did not show a significant influence. The interaction effect between SLE and family relationships was also significant. The $R^{2}$ change associated with the inclusion of the interaction term was .05 , and it could be considered a medium effect size (Cohen, 1992).

To interpret the interaction effect, three moderator variable groups were formed. Adolescents, $1 S D$ above and below the mean on QFR T1, were grouped into high and low quality. Adolescents with a mean score were grouped into a medium quality group. Additional regression analyses were conducted separately for each group. To interpret the significant interaction effect, simple slope lines based on the three regression coefficients were plotted (Figure 1). Post-hoc probes (Holmbeck, 2002) revealed that the association between stressful events and externalizing problems were not significant for the high quality group $(t(96)=1.10, \mathrm{~ns})$. For medium and low quality groups, slopes were positive and stepper, revealing that adolescents experiencing more stressful life events presented more externalizing problems $(t(96)=3.96, p<.001$ (medium quality); $t(96)=6.81$, $p<.001$ (low quality)).

A second hierarchical regression tested longitudinal effects. Table 3 shows that after controlling for externalizing syndromes in $\mathrm{T} 1$, the main effects were significant for life events, but not for quality of family relationships. Interaction effects were also significant with a small effect size (.04), as indicated by a change in $R^{2}$. We followed the same procedure to interpret the interaction effects (Figure 2). Post-hoc analyses revealed that the occurrence of stressful life events was related to an increase in the number of externalizing problems from middle-to-late adolescence, but only for medium and low quality of family relationships groups $(t(96)=3.18, p<.01$ and $t(96)=6.22, p<.001)$. When family relationships were of high quality, stressful life events did not lead to a change in problem behaviors $(t(96)=0.15$, ns).

Another hierarchical regression was performed using internalizing syndromes in T2 as criteria, and gender, stressful life events, quality of family relationships, and the interaction term between these last two variables as predictors. In this case, the results were clearly different from those which existed when the criteria was externalizing syndromes. Thus, as shown in Table 4, only gender and occurrence of stressful life events showed a significant association with internalizing syndromes, with non-significant interaction effects, for which it can not be interpreted that supportive family relationships protect adolescents from emotional consequences of SLE. In addition, an autoregressive model was conducted to study the possible influences of these variables in the change in internalizing syndromes from T1 to T2. This analysis indicated that only one variable (i.e., occurrence of SLE) contributed to explain the change (Table 5). 
Table 2. Regression models predicting Time 2 externalizing problems.

\begin{tabular}{lcccc}
\hline Predictors & Beta & $t$ & Cumulative $R^{2}$ & $R^{2}$ change \\
\hline Gender & -.00 & -.02 & .01 & \\
Stressful events & $.34^{* * *}$ & 3.95 & $.16^{* * *}$ & $.16^{* *}$ \\
QFR T1 $^{\mathrm{a}}$ & $-.37^{* * *}$ & -3.97 & $.26^{* * *}$ & $.10^{* *}$ \\
Stressful events $\times$ QFR T1 & $-.22^{*}$ & -2.53 & $.31^{* * *}$ & $.05^{*}$ \\
\hline
\end{tabular}

$N=100 .{ }^{*} p<.05 ; * * p<.01 ; * * * p<.001$.

${ }^{\text {a }}$ Quality of family relationships in Time 1 .

\section{Discussion}

The present study used a longitudinal design to examine the buffering effect of family relationships on the linkage between stressful life events and internalizing and externalizing symptoms of adolescents. We found that high quality parent-adolescent relationships, such as those characterized by cohesion, communication, affection, and monitoring, protected boys and girls against the negative consequences of stressful life events on externalizing, but not on internalizing symptoms. The adolescents who enjoyed good relationships with their parents during mid-adolescence did not increase their externalizing problems in late adolescence as a consequence of the occurrence of stressful life events. However, these stressors did lead to an increase in the number of externalizing problems when the family relationships were of a middle quality or, especially when the family relationships were of a low quality. Risk and resilience research has proposed family support as one of the main factors that protect children against the adverse effects of stressors (Luthar, 2006; Masten, 2001), and a handful of studies have underscored the protective or buffering effect of responsive and supportive early family environments on child and adolescent adjustment (Jackson \& Warren, 2000; Murberg \& Bru, 2004; Quamma \& Greenberg, 1994; Yates,

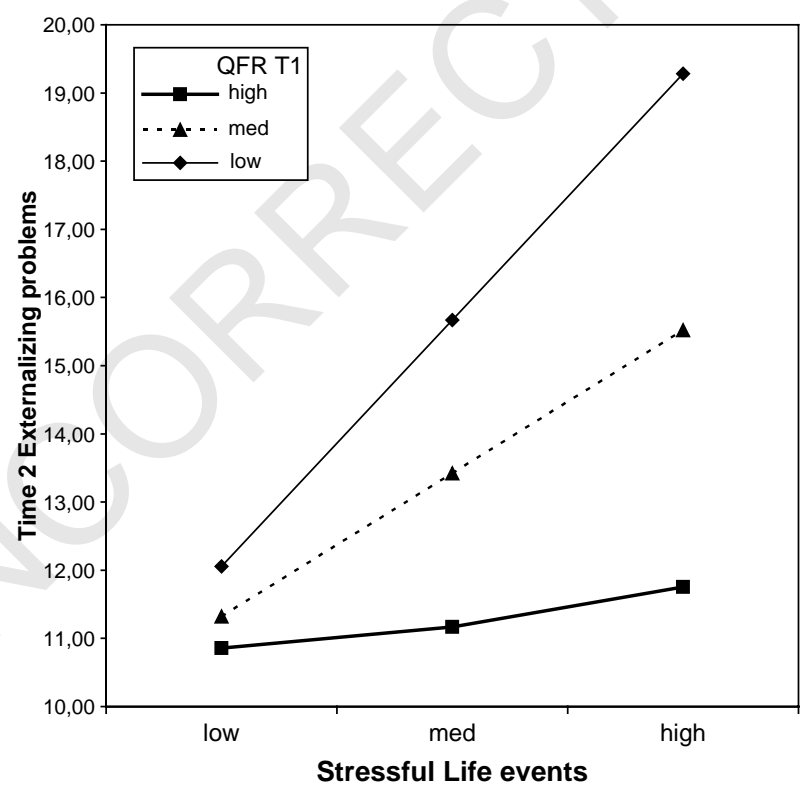

Figure 1. Interaction effects of stressful life events and quality of family relationships in Time 1 (QFR T1) on externalizing problems in Time 2. 
A. Oliva et al.

Table 3. Autoregressive model predicting Time 2 externalizing problems.

\begin{tabular}{lcccc}
\hline Predictors & Beta & $t$ & Cumulative $R^{2}$ & $R^{2}$ change \\
\hline Externalizing Time 1 & $.56^{* * *}$ & 6.66 & $.42^{* * *}$ & \\
Gender & -.03 & -.44 & $.42^{* * *}$ & .00 \\
Stressful events & $.23^{* *}$ & 3.20 & $.49^{* * *}$ & $.07^{* *}$ \\
QFR T1 & -.08 & -.96 & $.49^{* * *}$ & .00 \\
Stressful events $\times$ QFR T1 & $-.20^{* *}$ & -2.7 & $.53^{* * *}$ & $.04^{* *}$ \\
\hline
\end{tabular}

$N=100 .{ }^{*} p<.05 ; * * p<.01 ; * * * p<.001$.

${ }^{\text {a }}$ Quality of family relationships in Time 1 .

Egeland, \& Sroufe, 2003). There is less empirical evidence regarding the protective effect of parent-adolescent relationships during middle or late adolescence, which grants a higher value to our results, as they indicate that supportive parent-adolescent relationships continue to have a protective effect on behavior adjustment, at least for Spanish adolescents. Moreover, we should point out that quality of family relationships were deeply evaluated, since several instruments, with a total of 66 items, were used to collect information about different family dimensions. This is not an usual method since many studies are limited to include some items referred to parental support. The fact that interaction effects are especially difficult to detect because of the low statistical power in most studies (Frazier, Tix, \& Barron, 2004; McClelland \& Judd, 1993) increases the interest of our findings, mainly if we consider that we have used a longitudinal design to test moderator effects, something that has been less frequent.

Although in this study, some variables that might explain the buffering effect of parental support were not assessed, there is empirical evidence on the mediating role that

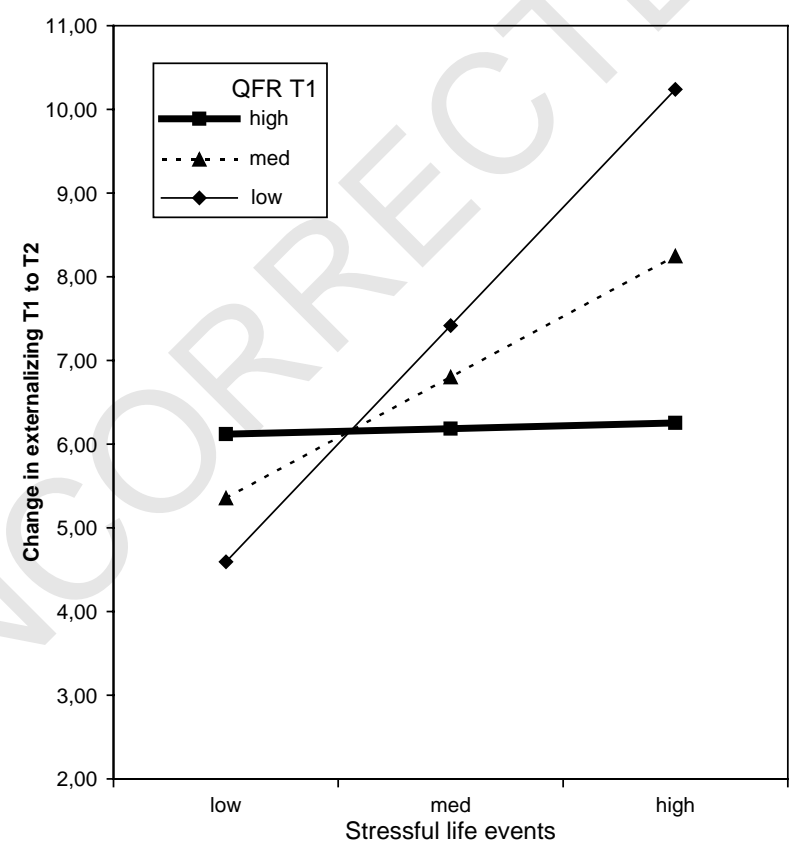

Figure 2. Interaction effects of stressful life events and quality of family relationships in Time 1 (QFR T1) on changes in externalizing problems from Time 1 to Time 2. 
Table 4. Regression models predicting Time 2 internalizing problems.

\begin{tabular}{lcccc}
\hline Predictors & Beta & $t$ & Cumulative $R^{2}$ & $R^{2}$ change \\
\hline Gender & $.26^{* *}$ & 2.65 & $.05^{*}$ & \\
Stressful events & $.27^{* *}$ & 2.86 & $.13^{* *}$ & $.08^{*}$ \\
QFR T1 & -.11 & -1.11 & $.14^{* *}$ & .01 \\
Stressful events $\times$ QFR T1 & -.12 & -1.24 & $.15^{* *}$ & .01 \\
\hline
\end{tabular}

$N=100 .{ }^{*} p<.05 ; * * p<.01 ; * * * p<.001$.

${ }^{\mathrm{a}} \mathrm{Quality}$ of family relationships in Time 1 .

coping strategies may play in the relationship between the perceived or received social support and the adjustment of the adolescent (Calvete \& Connor-Smith, 2006; Holahan, et al., 1997). It is probable that this support could influence the perception that adolescents have of potentially stressful situations, increasing their confidence in the resources they have to cope with, and reducing their emotional impact or preventing emotional disturbances from turning into externalizing problems (Lakey \& Cohen, 2000).

In contrast to what occurred with externalizing problems, interaction/buffering effects of family relationships on internalizing problems were not detected, which might be due to the relative stability shown by internalizing symptoms from $\mathrm{T} 1$ to $\mathrm{T} 2$, and also due to its dependence on other factors rarely linked to social support, such as the emotional regulation strategies that adolescents use to cope with distress (Nolen-Hoeksema, 1994).

Neither of the main effects of family relationships was significant. We found significant main effects on adolescent adjustment both of stressful life events and of family relationships. While life events were associated with an increase in externalizing and internalizing problems, the quality of family relationships was only related to externalizing problems, which coincides with results reported by Reitz, Dekovic, and Meijer (2006). Although research with children and young adolescents underlines the importance of parenting for emotional adjustment, our study focused on middle and late adolescence. The lack of a significant association between quality of family relationships and emotional problems can be attributed to the family influences during this period in competition with other types of influences, such as peer relationships, that represent a very powerful predictor of internalizing problems and self-esteem (Helsen et al., 2000; Scholte et al, 2001; Young, Berenson, Cohen, \& García, 2005).

Our results coincide with other studies that have found evidence for a connection between the experience of stressful life events and adolescent internalizing (Aseltine et al., 1994; Kraaij et al., 2003; Leadbeater et al., 1999; Murberg \& Bru, 2004; Rafnsson et al., 2006) and externalizing problems (Aseltine, Gore \& Gordon, 2000; Hoffmann \& Su, 1997; Kim et al., 2003), although there are longitudinal studies that have not found a relationship

Table 5. Autoregressive model predicting Time 2 internalizing problems.

\begin{tabular}{lcccc}
\hline Predictors & Beta & $t$ & Cumulative $R^{2}$ & $R^{2}$ change \\
\hline Internalizing Time 1 & $.69^{* * *}$ & 9.06 & $.54^{* * *}$ & \\
Gender & .01 & .19 & $.54^{* * *}$ & .00 \\
Stressful events & $.16^{*}$ & 2.29 & $.56^{* * *}$ & $.02^{*}$ \\
QFR T1 & .05 & .72 & $.56^{* * *}$ & .00 \\
Stressful events $\times$ QFR T1 & -.09 & -1.3 & $.57^{* * *}$ & .01 \\
\hline
\end{tabular}

$N=100 .{ }^{*} p<.05 ; * * p<.01 ; * * * p<.001$.

${ }^{\text {a }}$ Quality of family relationships in Time 1. 
between occurrence of stressful events and adolescent symptomatology some time later (Seiffge-Krenke, 2000). There are also abundant empirical evidences regarding the influence of parenting on adolescent behavioral problems (Perkins \& Borden, 2003), although the studies that employ a prospective methodology, as in our case, are more scarce (Steinberg, 2001).

Only one gender difference was significant (a higher incidence of internalizing symptoms in girls). This finding coincides with the results of the research performed so far, indicating that gender differences emerge in early adolescence and increase dramatically from middle to late adolescence, with girls showing during this period more anxiety and depressive symptoms (Leadbeater et al., 1999; Nolen-Hoeksema, 1994).

Some parents consider that they play a less important role when their children arrive in middle adolescence, so they relax their involvement and support. On the contrary, our study highlights the important role that family relationships seem to play in the behavioral adjustment of late adolescents, protecting them against some negative consequences of stressful life events when they are supportive. These results have, in our opinion, a clear practical interest for intervention, as they suggest the relevance of informing parents about their influence on adolescent well-being, and supporting them, through resources, such as parent education, which can help them to improve their parenting style by increasing their level of communication, affection, and monitoring. In a more favorable family context, boys and girls could show themselves more resistant to the negative effects of some adversities that are relatively frequent in adolescence.

Our study has some limitations. First, we assessed stressful life events in a retrospective way at the end of adolescence. This measure can be biased by the same variables that it pretends to predict, that is externalizing and internalizing problems, because these problems could have generated some stressful events (Cole \& Maxwell, 2003). In addition, all the informations were measured by self-reports completed by the adolescents. Even when the adolescent's perception of family relationships is less biased by social desirability than the parent's perception (Gonzales, Cauce, \& Mason, 1996), using only one reporter may have caused some bias because this practice creates a common method variance problem that may increase the size of the correlations among the variables. It would be important for future studies to employ other sources of information, such as parents, and to collect information about the occurrence of stressful life events in different waves of data collection rather than only in the last wave. Moreover, considering the protective role played by supportive family relationships, it is probable that other relations such as romantic or peer relationships, could also have similar effects. So, it would be really interesting to study the protective effect of these relationships on adolescent emotional and behavioural adjustment.

Another limitation was the reduced size of the sample used in the study that makes difficult the generalization of our findings.

In spite of these limitations, this study is important because, using longitudinal data, the protective role played by family relationships on the association between stressful life events and externalizing problems during middle and late adolescence has been demonstrated.

\section{References}

Abad, J., Forns, M., Amador, J.A., \& Martorell, B. (2000). Youth self report fiability and validity in an adolescent sample. Psicothema, 11(4), 1003-1011.

Achenbach, T.M. (1991). Manual for the child behavior checklist/4-18 and 1991 profile. Burlington, VT: University of Vermont Department of Psychiatry. 
Aiken, L.S., \& West, S.G. (1991). Multiple regression: Testing and interpreting interactions. Newsbury Park, CA: Sage.

Arbuckle, J.L. (2003). Amos 5.0: Update to the Amos user's guide. Chicago, IL: Smallwaters Corporation.

Aseltine, R.F., Gore, S., \& Colten, M.E. (1994). Depression and the social developmental context of adolescence. Journal of Personality and Social Psychology, 67, 252-264.

Aseltine, R.F., Gore, S., \& Gordon, J. (2000). Life stress, anger and anxiety and delinquency: An empirical test of general strain theory. Journal of Health and Social Behavior, 41, 256-275.

Belsley, D.A. (1991). Conditioning diagnostics: Collinearity and weak data in regression. New York: Willey \& Sons.

Calvete, E., \& Connor-Smith, J.K. (2006). Perceived social support, coping, and symptoms of distress in American and Spanish students. Anxiety. Stress and Coping, 19(1), 47-66.

Cohen, J. (1992). A power primer. Psychological Bulletin, 112, 155-159.

Cohen, S., \& Wills, T.A. (1985). Stress, social support and the buffering hypothesis. Psychological Bulletin, 98, 310-357.

Cohen, S., Kessler, R., \& Underwood Gordon, L. (Eds.). (1995). Measuring stress: A guide for health and social scientists. New York: Oxford University Press.

Cole, D.A., \& Maxwell, S.E. (2003). Testing mediational models with longitudinal data: Questions and tips in the use of structural equation modeling. Journal of Abnormal Psychology, 112, 558-577.

Collins, W.A., \& Laursen, B. (2004). Parent-adolescent relationships and influences. In R.M. Lerner \& L. Steinberg (Eds.), Handbook of adolescent psychology (pp. 331-361). NJ: Willey.

DuBois, D.L., Felner, R.D., Meares, H., \& Krier, M. (1994). Prospective investigation of the effects of socioeconomic disadvantage, life stress, and social support on early adolescent adjustment. Journal of Abnormal Psychology, 103, 511-522.

Dumont, M., \& Provost, M.A. (1999). Resilience in adolescents: Protective role of social support, coping strategies, self-esteem and social activities on experience of stress and depression. Journal of Youth and Adolescence, 28, 343-364.

Forjaz, M.J., Martínez, P., \& Cervera-Enguix, S. (2002). Confirmatory factor analysis, reliability and validity of a Spanish version of FACESIII. The American Journal of Family Therapy, 30, 493-449.

Frazier, P.A., Tix, A.P., \& Barron, K.E. (2004). Testing moderation and mediation effects in counseling psychology research. Journal of Counseling Psychology, 51, 115-134.

Ge, X. Lorenz, F.O. Conger, R.D. Elder, G.H. Jr., \& Simmons, R.L. (1994). Trajectories of stressful life events and depressive symptoms during adolescence. Developmental Psychology, 30, 467-483.

Gest, S.D., Neeman, J., Hubbard, J., Masten, A.S., \& Tellegen, A. (1993). Parenting quality, adversity and conduct problems in adolescence: Testing process-oriented models of resilience. Development and Psychopathology, 5, 663-682.

Gonzales, N.A., Cauce, A.M., \& Mason, C.A. (1996). Interobserver agreement in the assessment of parental behavior and parent-adolescent conflict: African-American mothers, daughters and independent observer. Child Development, 67, 1483-1498.

Gore, S., \& Aseltine, R.H. (1995). Protective process in adolescence: Matching stressors with social resources. American Community Mental Health Journal, 23, 301-327.

Grant, K.E., Compas, B.E., Stuhlmacher, A., Thurm, A.E., McMahon, S., \& Halpert, J. (2003). Stressors and child/ adolescent psychopathology: Moving from markers to mechanisms of risk. Psychological Bulletin, 129, 447-466.

Grant, K.E., Compas, B.E., Thurm, A.E., McMahon, S.D., \& Gipson, P.Y. (2004). Stressors and child and adolescent psychopathology: Measurement issues and prospective effects. Journal of Clinical Child and Adolescent Psychology, 33, 412-425.

Helsen, M., Vollebergh, W., \& Meeus, W. (2000). Social support from parents and friends and emotional problems in adolescence. Journal of Youth and Adolescence, 29, 319-335.

Herman-Stahl, M., \& Petersen, A.C. (1999). Depressive symptoms during adolescence: Direct and stress-buffering effects of coping, control beliefs, and family relationships. Journal of Applied Developmental Psychology, 20, 45-63.

Hoffmann, J., \& Su, S. (1997). The conditional effects of stress on delinquency and drug use: A strain theory assessment of sex differences. Journal of Research in Crime and Delinquency, 34, 46-78.

Holahan, C.J., Moos, R.H., Holahan, C.K., \& Brennan, P.L. (1997). Social context, coping strategies, and depressive symptoms: An expanded model with cardiac patients. Journal of Personality and Social Psychology, 72, 918-928. 
Holmbeck, G.N. (2002). Post-hoc probing of significant moderational and mediational effects in studies of pediatric populations. Journal of Pediatric Psychology, 27, 87-96.

Hoyle, R. (1995). Structural equation modeling: Concepts, issues and applications. Thousand Oaks, CA: Sage.

Jackson, Y., \& Warren, J. (2000). Appraisal, social support, and life events: Predicting outcome behaviour in school-age children. Child Development, 71, 1441-1457.

Jose, P.E., \& Kilburg III, D.F. (2007). Stress and coping in Japanese children and adolescents. Stress, Anxiety \& Coping, 20, 283-298.

Kagitcibasi, C. (1996). The autonomous-relational self: A new synthesis. European Psychologist, 1, $180-186$.

Kim, K.J. Conger, R.D. Elder, G.H. Jr. , \& Lorenz, F.O. (2003). Reciprocal influences between stressful life events and adolescent internalizing and externalizing problems. Child Development, $74,127-143$.

Kraaij, V., Garnefski, N., de Wilde, E.J., Dijkstra, A., Gebhardt, W., Maes, S., et al. (2003). Negative life events and depressive symptoms in late adolescence: Bonding and cognitive coping as vulnerability factors? Journal of Youth and Adolescence, 32, 185-193.

Lakey, B., \& Cohen, S. (2000). Social support theory and selecting measures of social support. In S. Cohen, L.U. Gordon \& B.H. Gottlieb (Eds.), Social support measurement and interventions: $A$ guide for health and social scientists (pp. 29-52). New York: Oxford.

Lamborn, S.D., Mounts, N.S., Steinberg, L., \& Dornbush, S.M. (1991). Pattern of competence and adjustment among adolescents from authoritative, authoritarian, indulgent and neglectful families. Child Development, 62, 1049-1065.

Lamothe, D., Currie, F., Alisat, S., Sullivan, T., Pratt, M., Pancer, S.M., et al. (1995). Impact of a social support intervention on the transition to university. Canadian Journal of Community Mental Health, 14, 167-180.

Lazarus, R.S., \& Folkman, S. (1984). Stress, appraisal and coping. New York: Springer.

Leadbeater, B.J., Kuperminc, G.P., Blatt, S.J., \& Hertzog, C. (1999). A multivariate model of gender differences in adolescents' internalizing and externalizing problems. Developmental Psychology, 35, $1268-1282$.

López, S. (2002). The FACES II in the evaluation of family cohesión and adaptability. Psicothema, $14,159-166$.

Luthar, S.S. (2006). Resilience in development: A synthesis of research across five decades. In D. Cicchetti \& D.J. Cohen (Eds.), Developmental psychopathology: Risk, disorder, and adaptation (Vol. 3 (2nd ed), pp. 739-795). New York: John Wiley \& sons.

Luthar, S.S., \& Zelazo, L.B. (2003). Research on resilience: An integrative review. In S.S. Luthar (Ed.), Resilience and vulnerability: Adaptation in the context of childhood adversities (pp. 510-549). New York: Cambridge.

Martínez-Pampliega, A., Iraurgi, I., Galíndez, E., \& Sanz, M. (2006). Family Adaptability and Cohesion Evaluation Scale (FACES): Development of a 20 items version in Spanish. International Journal of Clinical and Health Psychology, 6, 317-338.

Masten, A. (2001). Ordinary magic: Resilience processes in development. American Psychologist, 56, 227-238.

McClelland, G.H., \& Judd, C.M. (1993). Statistical difficulties of detecting interactions and moderator effects. Psychological Bulletin, 114, 376-390.

Murberg, T., \& Bru, E. (2004). Social support, negative life events and emotional problems among Norwegian adolescents. School Psychology International, 25, 387-403.

Nolen-Hoeksema, S. (1994). An interactive model for the emergence of gender differences in depression in adolescence. Journal of Research on Adolescence, 4, 519-534.

Oliva, A., \& Arranz, E. (2005). Sibling relationships during adolescence. European Journal of Developmental Psychology, 2, 253-270.

AQ2 Olson, D.H., Portner, J., \& Lavee, Y. (1985). Family Adaptability and Cohesion Scale. University of Minnesota.

Perkins, D.F., \& Borden, L.M. (2003). Positive behaviors, problem behaviors, and resiliency in adolescence. In R.M. Lerner \& M.A. Easterbrooks (Eds.), Handbook of psychology: Developmental psychology (Vol. 6, pp. 373-394). New York: John Wiley \& Sons.

Parra, A., \& Oliva, A. (2002). Family communication and conflict during adolescence. Anales de Psicología, 18, 215-231. 
Quamma, J.P., \& Greenberg, M.T. (1994). Children's experience of life stressors: The role of family social support and social problem-solving skills as protective factors. Journal of Child Clinical Psychology, 23, 295-305.

Rafnsson, F.D., Jonsson, F.H., \& Windle, M. (2006). Coping strategies, stressful life events, problem behaviors, and depressed affect among Icelandic adolescents: A cross-cultural replication study. Anxiety, Stress and Coping, 19, 241-257.

Reitz, E., Dekovic, M., \& Meijer, A.M. (2006). Relations between parenting and externalizing and internalizing problem behaviour in early adolescence: Child behaviour as moderator and predictor. Journal of Adolescence, 29, 419-436.

Rice, P.L. (1999). Stress and health (3rd ed). Pacific Grove, CA: Brooks/Cole.

Rudolph, K.D., \& Hammen, C. (1999). Age and gender determinants of stress exposure, generation, and reactions in youngsters: A transactional perspective. Child Development, 70, 660-677.

Seiffge-Krenke, I. (2000). Causal links between stressful events, coping style, and adolescent symptomatology. Journal of Adolescence, 23, 675-691.

Scholte, R.H.J., Van Lieshout, C.F.M., \& Van Aken, M.A.G. (2001). Perceived relational support in adolescence: Dimensions, configurations, and adolescent adjustment. Journal of Research on Adolescence, 11, 71-94.

Steinberg, L. (2001). We know some things: Parent-adolescent relationships in retrospect and prospect. Journal of Research on Adolescence, 11, 1-19.

Steinberg, L., \& Silk, J.S. (2002). Parenting adolescents. In I. Bornstein (Ed.), Handbook of parenting: Vol. I. Children and parenting (pp. 102-133). Mahwah, NJ: Lawrence Erlbaum Associates.

Stoolmiller, M., \& Bank, L. (1995). Autoregressive effects in structural equation models: We see some problems. In J.M. Gottman (Ed.), The analysis of change (pp. 261-278). Mahwah, NJ: Lawrence Earlbaum Associates.

Tao, S., Dong, Q., Pratt, M.W., Hunsberger, B., \& Pancer, S. (2000). Social support: Relations to coping and adjustment during the transition to university in the People's Republic of China. Journal of Adolescent Research, 15, 123-144.

Yates, T.M., Egeland, B., \& Sroufe, L.A. (2003). Rethinking resilience: A developmental process perspective. In S.S. Luthar (Ed.), Resilience and vulnerability: Adaptation in the context of childhood adversities (pp. 243-266). New York: Cambridge.

Young, J.F., Berenson, K., Cohen, P., \& García, J. (2005). The role of parent and peer support in predicting adolescent depression: A longitudinal community study. Journal of Research on Adolescence, 15, 407-423.

Zegers, B., Larraín, M.E., Polaino-Lorente, A., Trapp, A., \& y Díez, I. (2003). Validity and reliability of the Olson, Russel and Sprenkle family cohesión and adaptability scale in diagnosing family functioning in the Chilean population. Revista Chilena de Neuropsiquiatría, 41, 39-54. 


\section{AUTHOR'S QUERY SHEET}

Author(s): A. Oliva et al. GASC 308395

Article title:

Article no:

Dear Author

The following queries have arisen during the editing of your manuscript and are identified on the proofs. Unless advised otherwise, please submit all corrections using the CATS online correction form.

AQ1 This reference [Gore and Aseltine (1996)] is not cited in the text. Please cite or delete it.

AQ2 Please provide city/state of the publisher. 